\title{
New Understanding of Time Based on the Concept of Areal Multitudes ${ }^{1}$
}

\author{
Pavel V. Poluyan* \\ "Eniseygeofizika" JSC \\ 66 Leningradskaya Str., Krasnoyarsk, 660034, Russia
}

Received 20.12.2014, received in revised form 06.02.2015, accepted 22.03.2015

\begin{abstract}
The article puts forward a new ontology of the Time of Nature based on the following statements: 1) there is a multitude that we call "Time"; 2) this multitude consists of an infinite number of individual elements that we call "Instants"; 3) all the elements of the given multitude have a following feature: if one element is REAL, all the other elements of the multitude are UNREAL; 4) we shall call the multitudes of such type "AREAL MULTITUDES." It was discovered that the elementary areal ratio is a logical law of contradiction: $A$ and NON-A form together an areal multitude of two elements. In other words, if A is real, NON-A is unreal, but we see that this NON-A does not disappear, because without it, A is logically impossible. Nevertheless, if A exists, NON-A does not exist in reality. Thus, $N O N-A$ exists only as a possibility, it is "areal." Formulating the law of contradiction, Aristotle, and all the logicians after him, constantly underlined the fact that $A$ and NON-A cannot be in the same ratio at the same time. We would like to rearrange accents: in our formulation AREALITY is a particular logical ratio that simulates the Time of Nature. An infinite multitude of instants of Time is an areal multitude, because reality of the Present instant makes all the other instants of this infinite multitude unreal. We determine that the infinite areal multitude is also the multitude of normalizations of the numerical axis and suggest it as a model of Time. The new model determines the Time order as a symbolic sequence where the instants are the symbols of normalizations represented as unequal, actual infinitesimals. This approach allows us to detect periodization related to the mathematical constant e (Euler's number) on the infinite multitude of Time. The given unconventional conclusion is indicative of appropriateness of the proposed model.
\end{abstract}

Keywords: time, model, infinity, reality, unreality, multitude theory.

Research area: philosophy.

\section{Introduction and Statement of the problem}

Augustine of Hippo was probably cunning when he said that he intuitively comprehended Time, butcouldnotputitintowords("Confessions", Book XI, Chapter XIV). The philosophertheologian used a rhetorical technique bringing the reader closer to the unusual conclusion of the subjectivity of the Time structure - Past, Present and Future. This conclusion helped to get out of the logical difficulties when only the Present is really existent and the Past and the Future are non-existent. "But how is the future diminished or consumed when it does not yet exist? Or how does the past, which exists no longer, increase, unless it is that in the mind in which all this happens

(C) Siberian Federal University. All rights reserved

* Corresponding author E-mail address: polyan2002@mail.ru 
there are three functions? For the mind expects, it attends, and it remembers; so that what it expects passes into what it remembers by way of what it attends to. Who denies that future things do not exist as yet? But still there is already in the mind the expectation of things still future. And who denies that past things now exist no longer? Still there is in the mind the memory of things past. Who denies that time present has no length, since it passes away in a moment? Yet, our attention has a continuity and it is through this that what is present may proceed to become absent", - sums up Augustine of Hippo. (“Confessions”, book XI, chapter XXVIII, 37). Thus, in spite of rhetorical self-derogation, St. Augustine managed to answer the question - "What is time?" However now, after 1600 years, his conclusions look more like sharpening of the problem of time rather than its rational solution.

In 1908, McTaggart analyzed the same problem in the article "The Unreality of Time" (McTaggart 1908, pp. 457-73) He noted that the positioning of events in time is interpreted in two ways: events and moments can be described according to the "before-after" values (B-series in McTaggart's terminology), or their relation to the present, past and future (A-series). At the same time McTaggart easily proves that the B-series does not embody anything specifically temporal: it is similar to the spatial distribution of points on the axis - closer/further with respect to a reference point. But the A-Series turns out to be a specific feature of Time with the given characteristics: Past, Present, Future. However McTaggart decides to reject the specifically temporal A-series as a non-scientific, metaphoric structure, since he discovers insurmountable logical difficulties associated with it. Firstly, the moment of Time as such should be determined by a more fundamental external way, because the three characteristics of the Past, the Present and the Future characterize moments by turns, which means away from the point. Secondly, McTaggart says that interpretation of a single event should be extended to the moments of Times: if the events happen, then a certain moment also "happens" when it becomes a moment of the Present. But here "bad infinity" occurs - a moment M coming from the future and acquiring the status of the "moment of the present" acts as the event itself and as the moment of this event that we just began to interpret as the occurred event at the same time. The third difficulty discovered by McTaggart is that any occurred event turns out to be the event of the present and the event of the past, which is a contradiction. McTaggart notes that the verb form "was" means that the moment $\mathrm{M}$ in the past, which itself EXISTS, has already happened. In other words, in the moment of the past there is a moment that, as an event, occurred in the present. But it is the same moment! Therefore, we must assert that a single moment $\mathrm{M}$ is characterized in two ways: in the past there is a moment that is present. This is indeed a contradiction following from the definitions of the A-series: the past is made up of moments, though it is not some abstract points-instants, but the full-fledged momentsevents - the events of the occurrence of the moment in the Present. McTaggart considered this contradiction insurmountable and indicative of meaninglessness of the A-series. The revealed dilemma is similar to that considered by Saint Augustine: there are "three tenses - the present of the past, the present of the present and the present of the future", however, since they all exist in the mind of the subject, Augustine did not see the absurdity here (in fact, it is for this purpose that he did subjectivation of the Past, the Present and the Future). This structuring of verb tenses also allowed the English-language authors to develop a special "logic of time" associated with language patterns (Prior 1968). However, these constructs were not perceived as simulation of the objective time, and yet it is a statement of such problem 
that can be seen in conclusions of McTaggart, if we interpret difficulties that he found not as insurmountable absurd, but as logical conclusions that need rational interpretation.

Instead, in the $20^{\text {th }}$ century, the main trend in the study of time was set by relativistic physics, where the A-series was generally excluded from consideration - it was replaced by the B-series that turned into an axis of a four-dimensional spacetime continuum. In this case, the infinite temporal dimension covered the Past and the Future at the same time, but the Present was identified with a symbolic reference point. Accordingly, the time formation associated with the concepts of the Past, the Present and the Future began to be understood just as words of the everyday language to express the irreversibility of physical phenomena at the macro level (Grunbaum 1964).

However, the problem of Time is still not solved. Not by accident the global model of the evolving Universe based on the equations of relativistic physics led to the concept of the "Cosmological arrow of time." This means that the demarcation of objectivity and subjectivity in the understanding of Time still remains acute. The $21^{\text {st }}$ century in physics began with opposition of two concepts in physics: the author of one concept denies the objectivity of Time (Barbour 1999), and in the other theory the scientific understanding of Time is declared as one of the most important tasks, without which the development of physics is impossible (Smolin 2013).

Of course, the second approach is more constructive - it formulates the problem and does not "sweep it under the carpet." Back in his days, speaking of Immanuel Kant who denied the objectivity of Time the Russian philosopher Alexey Losev noted wittily: "If we set a purpose of our philosophizing about time as the evidence of its subjectivity and, like Kant, assume that time is not a property of things and is not an objective reality, but something that our subjective consciousness brought into things, then, in this way, we will not resolve the question of the essence of time, because we will talk not about time itself, but about its particular origin using the given concept of time. And we must say to Kant that we should first define what time itself is, and only then consider whether it is subjective or objective. I do not know what time is: how can I say if it is subjective?" (Losev, 1990, p. 299).

We believe that the above features that were found in the structure of Time divided into the Past, the Present and the Future provide material for the construction of its adequate model. In other words, it is the difficulties that led Augustine to subjectivization of time and made McTaggart leave the time series out of the equation of analytical science that will allow us to formulate a fundamentally new model of Time and answer the question: "What is time itself?"

\section{Theoretical Framework and Methods}

As we have noted above, development of relativistic physics in the $20^{\text {th }}$ century led directly to two opposite results. On the one hand, having become one of the four axial measurements of the pseudo-Euclidean continuum, Time got spatialized, which means that it lost a specific time fluctuation. (It is even measured in meters because seconds are multiplied by the $\boldsymbol{i C}$ factoran imaginary unity with a constant of speed of light). On the other hand, cosmology raises an acute problem of direction of Time, because solutions of relativistic equations led to the conclusion of cosmogenesis - it turned out that the Universe changes regularly starting from the first moment of its occurrence (this event was called the "Big Bang"). Thus, the scientific worldview has experienced a real revolution: formerly rationalists took pride in that they do not need the "hypothesis of God" (it is unnecessary because the world is not created and exists forever), but 
non-classical science began to speak about "the birth of the universe" (although a reasonable cause of this creation is still left out of the equation of the scientific content). So the question "What was there BEFORE?" has been raised again. And the greatest scientists of our time do not consider it metaphysical (Penrose 2014). The above dilemma suggests that the spatial model of time is missing some important features of the temporal process.

The first scientist who began to criticize physics for spacing of Time was Henri Bergson, and then, in Russian philosophy, Semyon L. Frank and Aleksei F. Losev pointed to an abstract one-sidedness of this approach: in mathematical models of physics Time is geometrized and likened to a one-dimensional linear continuum - thus, it is deprived of its specific qualities associated with fluctuation. However, physical science is not confined to the Minkowski's theory of the spacetime continuum. Hans Reichenbach noted: "In the processes studied by thermodynamics, physics is interested in the problem of the passage of time; therefore, the assertion that physics supposedly "spaces" time is the result of a profound mistake. A specific nature of time that is different from the nature of space is reflected in the fundamental equations of physics" (Reichenbach 1956). Abstracting from the fact of the temporary formation and replacement of its motionless geometric linear scale to measure time intervals is by no means the only option for simulation of Time. G. Whitrow in "the Natural Philosophy of Time" (Whitrow 1961) pointed to the famous mathematician William Rowan Hamilton who proposed the creation of a theory of pure Time based on algebra considering geometry as a theory of pure space.

Hermann Minkowski said that space and time individually became empty fictions and only their unification retains an independent reality (Minkowski 1914). However, it is not considered that their unification is again the space (formally and mathematically), and the time axis is scaled in spatial terms. In other words, the space has kept its own characteristics, and the time lost them. Thus, we face a problem: either it is necessary to agree with the geometrical representation of time as one of the measurements of the 4-dimensional space-time continuum and accept this model as comprehensive or, considering this approach as a certain step closer to the truth, try to give a new model of time, where the time qualities would be taken into account.

In our opinion, the manifestation of subjectivity is not an illusion of the temporal process, but the simulation of the real-time process by means that cannot adequately express it. In our view, the question of geometric or algebraic methods of simulation of time is extremely important. It shows that significantly different results can be obtained with different simulation techniques - different understandings of what Time is. The traditional scientific methodology describes how a theoretical (formalmathematical) model is built on the basis of the experiment data. Then this model is compared with the object of the study, specified, modernized or rebuilt. It is important to emphasize that the simulation techniques (formal systems) also have some properties that limit the representation of the real object of the study making the model inadequate. Below we propose a model of Time based on the concept of the areal multitudes. This article presents the philosophical foundations and describes the qualitative features of this model.

\section{Discussion: new model of Time, areal multitudes}

In graphs and charts, Time is represented as a one-dimensional linear continuum of real numbers, where each coordinate point corresponds to a certain moment. In theoretical physics there are also exotic models, where moments form a countable multitude (this 
implies a discrete structure of time) or models, where a circular order of moments is acceptable. Obviously, all this is geometric representations and specific spatial images (in a circular order, a spatial model generally destroys the specifics of time that is logically determined through the order of moments - as noted by Alexander Zinoviev (Zinoviev 2010, p. 134). In this case, the semantic characteristics of space substitute the concept of Time. An alternative approach would be that where Time is modelled using relations typical for the passage of time, but not for the frozen spatial extension. We suggest this new model of time based on the new concept of "AREAL MULTITUDE", which is introduced as a result of the analysis of the structure of the Time of Nature.

The immanent property of the natural structure of Time (as shown by McTaggart) is the division of a single temporal flow into the Past, the Present and the Future. In addition, Time is usually represented as an infinite multitude of individual instants.

It seems it is not difficult to divide the entire multitude of instants into three parts: instants of the Present, instants of the Past and instants of the Future - as it is done in relativistic physics with the help of the so-called "light cone" establishing subordination of the causally related events. However, occurs a certain ambiguity (as was clear even to St. Augustine): there is no Future in reality (it has not YET come), and we talk about the existence of instants of the Past in a certain sense, because they are ALREADY gone. In other words, there is no past IN THE SENSE that there are no available physical states of the material world that have been before, and there is no future because the present state changes it will be replaced by other states. However, this interpretation hides substitution of concepts: the change of states of material systems is just an external indicator of the passage of time. In other words, the concept of time does not consist in that there are different states of material things, but in logical constructs - ANY MORE and NOT YET - clearly showing the very structure of time that should be taken as an object of the study. We need to learn the logical structure of time, but not reduce time to the change of physical states or senses, since any such reduction presupposes variability - suggests the logic of time.

On the other hand, as we have seen in McTaggart's work, the analytical examination of relations of the Past, the Present and the Future inevitably leads to semantic difficulties. We believe that these difficulties can be overcome by using the concept of areality.

Once again, let us set up the prerequisites. Firstly, time is an infinite multitude of instants. Secondly, the whole multitude of instants is ALWAYS divided into three submultitudes: the Past, the Present and the Future. Thirdly, only the instant of the Present really exists, while the instants of the Past do not exist ANY MORE and the instants of the Future HAVE NOT become existing YET. If a certain instant does not refer to the non-existing instants, it, nevertheless, could become present earlier, or it will become present later on because time passes. This will be fixed in the concept of an areal multitude: an element of the areal multitude is real when and only when all the other elements are not real.

And it is absolutely obvious for Time: an instant of the Present is real when all the other instants are carried out into unreality - the Past or the Future.

Let us point to the ambiguity. A definition of the areal multitude differs from the classical definition of a multitude where all the elements exist and are actually defined. Furthermore, the general notion implies that not only the instants of time can be presented as an areal multitude, but any other totality of elements as well. So where are they? 
It is easy to answer the first question: all the concepts appear in science to be specified and developed. In other words, the classical multitudes may remain in the frames of their definitions, but the areal multitudes are a different kind of totality: the areal multitude is a totality of elements where each element is real when and only when the other elements of the given multitude are not real. Areal multitudes, as well as the regular multitudes, may consist of an infinite or a finite number of elements, but the minimal number of areal multitude elements would be two.

Now let us answer the second question: Is there any specific multitude with inherent areality? Oddly enough, the ratio of areality in mathematics and logic can be seen quite often, it is just it has not been noticed before, it has not been considered as something special. In logic, this is due to the formal application of the strict disjunction "EITHER-OR", when some $\mathrm{A}$ and $\mathrm{B}$ are in a ratio of mutual exclusion. As for mathematics, we can find quite informative examples of areality.

In geometry, when we set up a Cartesian coordinate system for the three-dimensional space and mark the axes $\mathbf{O X}, \mathbf{O Y}, \mathbf{O Z}$ we usually note that depending on the order of the axes there are two possible variants - the "left" coordinate system and the "right" one. If we choose the "right" coordinate system, the "left" one is excluded, that is, here we have the ratio of areality. It is not taken into account considering that it is not a mathematical fact, but a subjective feature - arbitrary of denomination. Meanwhile, the famous mathematician Nikolai N. Luzin and the Academician Vladimir I. Vernadsky discussed this issue: "The question of whether there are two Euclidean spaces, one of which is levorotatory and the other is dextrorotatory. And what perfect axioms can characterize both spaces?" (Luzin 1993). Obviously, the "left" and the "right" variants of the three-dimensional space axes are in the areal ratio.

We find another example of the areal ratio in the Dedekind's definition of the continuity of a one-dimensional continuum through section: "We can say that each number "a" produces one or, in fact, two sections, that, however, will not be considered as essentially different; moreover, this section has such a property when among numbers of the first class there is the biggest one, or among the second-class numbers there is the smallest one." (Dedekind 1923, p. 19) That is, the number producing section should be assigned either to the left side of the numeric axis (as the biggest number in this "lower" class) or to the right side (as the smallest number in the "upper" class). Both variants are the same Dedekind sections and are in the areal ratio (when one is accepted, another one is excluded), but mathematicians do not focus on the existence of the two alternatives ("will not be considered") considering it immaterial.

And finally, it is particularly noteworthy that we find the areal ratio in the very foundation of classical logic - the law of contradiction. "It is impossible that the same thing at the same time was not peculiar to one and the same thing in the same respect" - determines Aristotle in "Metaphysics" (Aristotle, 1976, p. 125). Here the areal multitude is the one with two assertions consisting of the proposition $\mathrm{A}$ and its negation NON-A. If A is true (real), its negation, NON-A, is not true (not real); but if non-A is true, then its negation, $\mathrm{A}$, is not true (not real). Here the areality is obvious and not accidental, as the logical law of contradiction states that $\mathrm{A}$ and non-A cannot be true at one and the same time. Thus, we see that the ratio of areality is not some spontaneous fabrication, but it is implicitly put into the principal law of logic, the law of contradiction. But having found out it in its explicit form, we come up with the following summary: an areal multitude of two propositions, A and non-A, has 
turned out to be the simplest variation of a more complicated ration.

In all of these examples the areal multitudes are the finite multitudes consisting of two mutually exclusive variants, but for us, it would be interesting to find an infinite areal multitude in order to simulate time consisting of an infinite multitude of instants. So we find this multitude.

To operate with numbers people use the so-called positional number systems. It is an indisputable fact. Typically, a decimal system is used, and in computer studies it is a binary system, but it is clear that any number greater than one can become a basis of the positional number system (although an irrational number is not appropriate in this role). It is obvious that there is an infinite number of such systems and they are alternative. Number systems are the substance of construction created for a simplified number designation. It is an algorithm of formation of a unique name for each real number. And although a multitude of number systems is not a mathematical, but cultural and civilizational fact, there is a certain areal subtext here. Behind multiplicity of the positional systems there is a certain significant mathematical property that can be understood as objectively given (according to the philosophy of mathematical realism “mathematical Platonism”). (Putnam 1983, p. 74). We can easily find this property that is going to be called "normalization of the numeric axis" here. This is, again, a mathematical fact considered to be trivial, but the analysis can detect a sign of areality in it. All positional systems are based on the fact that a certain basic number is allocated for each of them and is taken for as the number of the first rate, and further numeration is carried out so that new numbers are recalculated for a specified number of times. Thus, 10, 100, 1000, etc. form a decimal system. In other words, all of the number systems are based on the property of NORMALIZATION inherent in the numeric axis - from one to infinity any of the numbers can be taken as a new unity. A multitude of normalizations of the numeric axis is a multitude of variants of the unit measures that can be set on a one-dimensional linear continuum. And here, if one normalization is selected (made real), all the other normalizations are unreal.

Normalizing the numeric axis is not marked as a special subject of the mathematical study, for it is regarded as trivial. Indeed, what could be more ordinary than a numeric axis with points that are mapped by the real numbers? We say: this point on a numeric axis is a unity and, respectively, set 2 , 3, 4 and so on. For the implementation of the other normalization, for example, " 3 " becomes a unity, and "l" from the previous normalization (the one that is now superseded in areality) becomes the number $1 / 3$. It is this trivial operation that plays a major role in the construction of the positional number systems. Accordingly, all the possible normalizations of the numeric axis form a kind of an infinite multitude, but on a given multitude there is always a certain elementary unity - a normalization taken as real, and the rest of the normalizations are taken into areality. But they all form a single multitude (we can move from one normalization to another using a normalization factor).

So, it turned out that a multitude of normalizations is an areal multitude. But we started with determining Time as the areal multitude as well. We identified submultitudes of instants of the Past, the Future and the Present, and in order to describe their relations we had to introduce the ratio of areality. If the areal multitude of normalizations is a special mathematical structure, why do not we take it as a basis for a model of Time? Thus, it is proposed to identify the areal multitude of normalizations of the real numeric axis with chronometrics of natural time. Such identification, in our opinion, is constructive and yields interesting 
conclusions. Time now is modelled not just as a one-dimensional continuum of real numbers, but it finds its theoretical conception, its model, in a much more complex structure.

The proposed model needs conceptualization and further development. We hope that this approach will be interesting to logicians and mathematicians. Here we have introduced some kind of innovation - the principle of areality and areal multitudes that were used for simulation of time. Even without regard to such a model the areal multitudes are interesting objects for study. For example, it is easy to find a ratio opposite of areality, which is a case of the logical connection between individuals "if there is A, there is also B." You can also introduce conjugate areal multitudes: for the areal multitudes of two elements the definitions "if A exists, then B does not exist" and "if A does not exist, then B does not exist" are equivalent - that is, one and the same multitude is determined in both cases. But it is different for the infinite areal multitudes. The statement "if one element exists, all the others do not exist" generates an areal multitude, but the opposite ratio "if one element does not exist, all the others exist" generates another multitude (in our model, it is not the time that is equivalent to it, but a certain spatial continuum with an excluded point). Thus, the study of areal objects is an independent task. However, it is followed by an unexpected conclusion: we have no right to introduce the direct identification of the areal multitude of normalizations with the areal multitude of instants of time; our areal multitude is only a primary mathematical structure to construct a more adequate model of Time. Let us outline the next steps.

So, we take a multitude of normalizations as only the first stage of building a model of Time. The multitude of normalizations is a mathematical structure that simulates relation between the instants of the Past, the Present and the Future.
Earlier we examined the traditional model of time as a linear one-dimensional continuum and noted that in that model time is spatialized: structural properties of time associated with the transition of the Future into the Past through the Present are excluded. That is why we started looking for a new model, but won't it be the same story? In other words: have not some important features of Time moved to the background? Indeed, the space-like model was good because it gave a pictorial presentation of such a property of time flow as duration - on a linear scale there are segments - intervals of time. Such a T scale coupled with spatial dimensions allowed building visual timetables and determining the operation of differentiation $\mathrm{dx}$ by $\mathrm{dt}$. But in the new model of Time, identification with a multitude of normalizations does not seem to give us such opportunities: we just showed how the choice of one normalization as the main one displaces all other possible normalizations into areality, as well as an instant of the Present suggests that all the instants of the Past and the Future are unreal. But how can we form out of these normalizations the durations of time that are usually compared with each other? This is the issue we have to solve.

The new model has another drawback. Real Time sets a sequence of instants structured by the principle of "earlier-later". For normalizations, there is their own structuring by the principle of "more-less" - in compliance with a magnitude of numbers taken as a norm-unity. So the following question arises: should we identify the "moreless" on the multitude of unities-normalizations with the "earlier-later" typical for the temporal orientation? When answering it, we find an interesting property of the studied model: it is clear that the alignment of normalizations in a certain series by the principle of "more-less" is only one variant of construction of the sequence of normalizations, and in general, there can 
be infinitely many of such variants. In order to correlate a number of normalizations with a series of instants, it is only required that normalizations in the construction of a series are not repeated. The orientation is set by the order of normalizations in sequence, but a specific implementation of the order of normalizations in this sequence can be of any nature. In other words, in real Time takes place the random alignment of normalizations in a series in an infinite number of variants - a kind of an infinite symbol sequence is realized this way (like a DNA chain, only not from a finite number of elements-symbols, but from an infinite set of elements).

Bioinformatics studies mathematical aspects of symbol sequences, so the application area for this kind of research opens in our time model. It is generally good, but in the real-time flow, which we took to model, there is the stability of the Past where all the instants-normalizations have already aligned in a series (i.e., there is already a stable symbol sequence of normalizations, which lined up from the eternity of the Past to this instant of the Present). But the Future is revealed as endless variants of addition of symbolsnormalizations (where, in fact, we find areality: implementation of one sequence excludes all the others). Thanks to the identification of moments of time with normalizations the instants acquired certain individuality: transformation of a given instant into the moment of the Present makes it a unique event (in full accordance with the statements of McTaggart), but in our model, this means a receipt by an instant of a certain place in the order of normalizations as a sort of a symbol sequence.

Another aspect of the model: we assume that any moment of time is not just a point on the numeric axis, but a normalization. The transition to the next instant of time is not a jump into an adjacent point on the axis (a coordinate point in the same normalization), but the transition to a point that is "adjacent", but represented in another normalization. Then the real time axis of the Past that "has already gone" is an uninterrupted continuum consisting of points, but radically different from the usual real axis: all points belong to different normalizations, and (as we have already defined above) the implementation of one normalization in the form of an instant of time leads to the exclusion of the possibility to implement the same normalization on the axis of the Future. If an instant has already been the Present, it means that a certain normalization has already been implemented and cannot be implemented again, because it would mean that the same instant occurred once again. This defines the net time, and it would be funny, if the same instants could occur, and the events "in them" could be new every time.

In general, these features allow us to see a non-trivial property of our model of time: in the linear space-like model, time is presented as a certain unfilled duration - in the form of instants as empty cells that can be filled with events in different combinations. Now we have identified the same combinatorics as an immanent property of the instants of time themselves. In other words, it is the net Time arranged as an infinite multitude of variants for positioning of instants, each of which is like an individual event because it is individualized in the identification with one unique normalization.

We have found that building a series of normalizations is a symbol sequence, a mathematical object studied in bioinformatics, where the specific methods of analysis are practiced. This analogy will help to answer the question of how duration may be formed from normalizations - above we noted that the comparison of durations of periods of time that was given by the spatial model remains uncertain for the areal time model. What is the difficulty? The spatial model of time allowed us to compare 
durations of time intervals as durations of spatial segments, and in the areal model of time we find intervals composed of the infinite series of normalizations instead of metrizable segments, but, at this, each point-normalization is rather just a name-symbol than a certain duration.

Indeed, a multitude of normalizations forms from symbols-numbers, each of which represents a certain normalization - a selected unity that is different from another selected unity. In one case, number $\mathrm{A}$ is taken for a norm-unity and in the other - number B, etc. At this, the numbers act not as the amounts, but as the names of normalizations. It is clear that quantitative characteristic is lost in the process of normalizing: in all the cases, a norm is equal to a unity. If we, for example, took up to compose duration from these norms-unities maintaining their values set on the numeric axis, we would obtain an absurd result as arbitrary symbol sequences, sequences of random numbers, for example, $1+3+50+2345+\ldots$ etc. But in such series, obviously, the unity is a norm, and if we select an adjoined three, we have to rewrite the entire series of summands, and this will give a totally different symbol sequence. In other words, the question arises: what symbols of normalizations can be used here - so that the selected order of these symbols would correspond to the entire multitude of normalizations, but not some single normalization taken as a normunity?

We have previously examined this situation in the publication "The Value of the Name" at the conference "Philosophy of Mathematics, topical issues. Proceedings of the International Scientific Conference, June 15-16, 2007, Moscow State University" (Poluyan 2007, p. 268). And earlier in the publication in English at the International Scientific Conference "Non-standard Methods and Applications in Mathematics", Italy, University of Pisa (Poluyan 2006). There we determined that an infinite multitude of equal unities can be named using actual infinitesimals that are not equal to each other. As we know, there is a so-called non-standard mathematical analysis that differs from the standard one in that the infinitesimals occur not through the process of taking the limit, but they are set actually. The standard analysis reveals that a significant segment can be divided into an infinite multitude of equal infinitesimals, and the creator of the non-standard analysis Abraham Robinson stated that "limitation by partitioning into intervals of equal length is too artificial. We will find a methodology that will allow us to consider more general subdivisions." (Robinson, 1967, p. 341). Our case - Time Modelling - corresponds precisely to this approach. Indeed, we do not take into account the finished spatial duration that can be split into an infinite number of infinitesimals. We do the opposite: a certain time interval is composed of infinitesimals that are unequal and collected randomly (each actual infinitesimal is a symbol for a single unit normalization forming a continuum of the real axis, where the unities are set in a specific normalization), but in general, a series of such unequal actual infinitesimals is a long duration.

With this approach we achieve the goal: any time interval really becomes a symbol sequence composed of names, each of which represents a unity of a certain numerical normalization, but since each such name in effect is an actual infinitesimal, their infinite set forms an interval of a quite significant duration. And vice versa: any time interval is composed of the infinite number of $\boldsymbol{d} \boldsymbol{t}_{\boldsymbol{i}}$ that are unequal to each other (hence a slightly modified definition of instantaneous velocity, but this issue should be considered separately).

So, we gave a tentative answer to the question: whether it is possible to determine the duration of the time interval, if Time is understood according to the model of the areal multitude of normalizations. We have proposed here some 
kind of innovation: the possibility to name equal unities with the help of actual infinitesimals, because if there is an infinite multitude of equal unities we can attribute a unique name in the form of an infinitely small part of the unity to each of them. (In this case, quantitative equity remains, because $\mathbf{1}+\mathbf{d} \mathbf{x}_{\mathbf{i}}=\mathbf{1}+\mathbf{d} \mathbf{x}_{\mathbf{j}}$, but there will be no identical equation because the names of these unities will be different - this corresponds to an algebraic expression $\mathrm{A}=\mathrm{B}$ ). The methodological difference from the conventional understanding of the algebraic record is that we do not consider different names of equal unities ("A", "B" and so on) as subjective introduction made for the convenience of calculation, but regard them as an immanent property that is objectively inherent in mathematical objects. This innovation could be considered arbitrary and artificial, but it allows us to get a non-trivial mathematical result directly related to the model of Time.

Let us examine the series presenting a usual abstract number that increases with each step by a unity: $\mathbf{1}+\mathbf{1}+\mathbf{1}+\mathbf{1}+\ldots$. We propose to name all the unities of this series and take the actual infinitesimal as the name for each of the given unity. In order to pass to the limit, we begin with a series of $\mathrm{N}$ unities with a specific name through the partitioning of the given unity into $\mathrm{N}$ parts. Let us assume that the first name is the $\mathrm{N}^{\text {th }}$ of the original unity. Then the naming process here is simple: the first unity $=1$, the second $=\mathbf{1}+\mathbf{1} / \mathbf{N}$, the third $=(\mathbf{1}+\mathbf{1} / \mathbf{N})+(\mathbf{1}+\mathbf{1} / \mathbf{N}) / \mathbf{N}$, etc. That is, each right unity is the $(\mathbf{N}+\mathbf{1}) / \mathbf{N}$ from the left preceding unity, or each next unity differs from the previous one by its $\mathrm{n}^{\text {th }}$ part - up to a certain step N. Now we should direct $\mathrm{N}$ towards infinity. And here we come up with an amazing conclusion: in such construction of a series the last unity located on an infinite place, to which other unities came from the first unity in an infinite number of steps (all steps differed by infinitely small names), is greater than the first unity by $\boldsymbol{e}$ times. It is a well- known formula: the limit $(\mathbf{1}+\mathbf{1} \backslash \mathbf{N})^{\mathbf{N}}$, when $\mathbf{N}$ is directed to infinity.

What does this mean? It turns out that infinity can be structured: it is possible to set an infinite number of the named unities starting with the first unity and ending with the last one that is related to the value of the first step - a very specific FINAL STEP is revealed. It is defined as a step that is equal to the length of the first step multiplied by the mathematical constant $e$ (basis of the natural logarithm, the Euler's number). We can continue to go on with the infinity, but we managed to set on an infinite line a GREAT UNITY consisting of an infinite countable number of steps. The Euler's number sets periods on an infinite axis, which is its NATURALNESS, and it determines the real STRUCTURE OF THE INFINITY. And given the fact that the mathematical constant $\boldsymbol{e}$ constantly arises in mathematical calculations associated with times and periods, this coincidence is very important. The definition of a non-trivial structure of the infinity associated with the Euler's number shows constructiveness of the path we have chosen.

Here we determined a series for the abstract number increasing by a unity resulting in a sum of an infinite number of unities. It is logical to assume that, in this case, we have the model of metrization of not some abstract number, but the model of the net Time composed of unities, each of which are named. This finding needs further analysis, and we will publish the results of this analysis later.

\section{Conclusion}

Let us summarize the article.

For simulation of Time we proposed the following statements:

1. There is a multitude that we call "Time".

2. This multitude consists of an infinite number of individual elements that we call "Instants". 
3. All the elements of this multitude have a unique feature: if one element is REAL, all the other elements of the multitude are UNREAL.

4. We call the multitudes of this type AREAL MULTITUDES.

We used the ratio of AREALITY for modelling of Time, since structuring Time into instants of the Past, the Present and the Future is based on the ratio of areality, because the recognition of this instant as the moment of the Present suggests that all other instants of time are future or past, which means that they do not really exist, although they are areally present.

The new model of Time is faced with two challenges: firstly, it is necessary to somehow determine metrizability of the multitude of instants to discover the duration of periods of time and, secondly, to distinguish between the areality characteristic for the Future and the areality characteristic for the Past.

Apart from the obvious AREALITY inherent to the logic laws of "contradiction" and "excluded middle", we also find the ratio of AREALITY for some well-known mathematical objects. Such as a Dedekind section and mutually exclusive names of axes of a three-dimensional space - the "right" and the "left" coordinate systems. Also, the ratio of AREALITY is found on the multitude of normalizations of the numeric axis that lies at the basis of the multitude of positional number systems. We propose to take an infinite areal multitude of normalizations of the numeric axis as a basis for modelling of Time.

We find that each unique normalization can be taken as a representation of an instant of time so that the actualization of the instant as the moment of the Present should occur only once (moments are not repeated). Then the time series is composed of a unique symbol sequence of randomly alternating normalizations. The symbols of normalizations are the actually infinite small parts of data of the normalized unities from different unique normalizations. Thus, the duration of time intervals is not a standard partition of the unity into an infinite number of equal infinitesimals, but the non-standard composition of the unit length of unequal actual infinitesimals understood as symbols of different normalizations. Therefore, at a new level we return to a one-dimensional continuum as representation of the Time duration. At the same time, the use of names for an infinite series of unities allows us to structure infinity based on the Euler's number (the first element in a series is different from the last element in a series by $\boldsymbol{e}$ times). We concluded that such structuring of infinity is characteristic of the one-dimensional time continuum.

In general, the proposed model of Time is formulated here only at the level of the initial philosophical propositions; it is qualitative, descriptive and needs further development and a more precise definition.

\footnotetext{
The concept of areal multitudes was proposed for the first time by the author for time modeling in 2004 in the Proceedings of the International Conference "Numder, Nime, Relativity". (P.V. Poluyan, Time: areal multitudes and chronometrics, Proceedings of the International Scientific Meeting, Bauman Moscow State University, Physical Department, Moscow, 2004, P. 71-73), and a brief summary of this topic is presented in the Proceedings of the 23rd World Congress of Philosophy in Athens in 2013 (Pavel Poluyan, "A new model of time. Areal multitudes", Section 58: Philosophy of nature. 23rd World Congress of Philosophy. Philosophy as Inquiry and Way of Life. Abstracts. Athens 04-10 August 2013, Greek Philosophy Society \& FISP, University of Athens, School of Philosophy. P. 572-573.) In the present article the time model is significantly deepened and complemented with new results.
}

\section{References}

1. Aristotle. Sochineniya v chetyrekh tomakh, t.1 "Metafizika" [Works in four volumes, Vol. 1. "Metaphysics"]. Moscow: Publishing House "Mysl”, 1976.

2. Augustine of Hippo. Confessions, Book Eleven, Chapter XXVIII, 37.) 
3. Barbour J. The End of Time: The Next Revolution in our Understanding of the Universe. Oxford Univ. Press, 1999.

4. Dedekind Richard. Nepreryvnost $i$ irratsionalnyye chisla [Continuity and irrational numbers] Odessa, Publishing house "Mathesis", 1923.

5. Grunbaum A. Philosophical Problems of Space and Time. New York: Alfred A. Knopf, Inc. A British edition was published by Routledge \& Kegan Paul, London, 1964.

6. Losev A. Muzyka kak predmet logiki [Music as a subject of logic] in the book by A.F. Losev From early works (Moscow: Publishing House "Pravda", 1990).

7. Luzin N.N. Pisma V.I. Vernadskomu [Letters to V.I. Vernadsky] From "The Russian thought" magazine - publication by L.G. Antipenko, No.1-2, 1993.

8. McTaggart J.E., The Unreality of Time, Mind: A Quarterly Review of Psychology and Philosophy, vol. 17, 1908.

9. Minkowski H. Prostranstvo i vremya [Space and time], in the book "New ideas in mathematics. Coll. 5: Principle of Relativity in mathematics". SPb: Obrazovaniye, 1914).

10. Penrose R. Tsykly vremeni. Noviy vzglyad na evolyutsiyu Vselennoy [Cycles of time. A new look at the evolution of the Universe]. Moscow: Publishing House BINOM, 2014.

11. Poluyan P.V. Financial Quantum and Quantity of Name: Non-Standard Methods and Applications in Mathematics? Italy, University of Pisa, NSM, 2006. - http://www.dm.unipi.it/ nsm2006/ schedule.30.hyml

12. Poluyan P.V. Velichina imeni: Filosofiya matematiki, aktualnyye problemy [The value of the Name: Philosophy of Mathematics, topical issues]. Proceedings of the International Scientific Conference on June 15-16, 2007, Moscow State University, Moscow: Publishing House Savin S.A., 2007, p. 268.

13. Prior, A.N. Papers on Time and Tense, London: Oxford University Press, 1968.

14. Putnam H. Reference and Truth: Realism and Reason, Philosophical Papers. Vol. 3. Cambridge, 1983.

15. Reichenbach H., The Direction of Time. University of California Press Berkeley and Los Angeles, 1956.

16. Robinson A. Introduction to Model Theory and to the Metamathematics of Algebra, NorthHolland publishing company, Amsterdam, 1963.

17. Robinson A. Vvedeniye v teoriyu modelei i metamatematiku algebry [Introduction to the Model Theory and to the Metamathematics of Algebra], Moscow: Publishing House "Nauka", 1967.

18. Smolin L. Time Reborn: From the Crisis in Physics to the Future of the Universe. Kindle Edition, 2013.

19. Whitrow G.J. The Natural Philosophy of Time, Thomas Nelson and Sons Ltd London and Edinburgh, 1961.

20. Zinoviev A. Logicheskaya fizika [Logical Physics] Moscow: Publishing House URSS, 2010. 


\title{
Новое понимание времени
}

\section{на основе концепции ареальных множеств}

\author{
П.В. Полуян \\ $О А О$ «Енисейгеофизика»
}

Россия, 660034, Красноярск, ул. Ленинградская, 66

\begin{abstract}
Предложенановаяонтологияестественного Времени, основаннаянаследующихопределениях: 1) существует множество, которое мы называем «Временем»; 2) это множество состоит из бесконечного числа индивидуальных элементов, которые мы называем «Моментами»; 3) у всех элементов данного множества есть особенность: если один элемент РЕАЛЕН, все другие элементы множества НЕРЕАЛЬНЫ; 4) назовем множества такого типа «АРЕАЛЬНЫЕ МНОЖЕСТВА». Обнаружено, что элементарное ареальное отнотение это логический закон противоречия: утверждения А и HE-А образуют вместе ареальное множество из двух элементов. То есть если А реален, НЕ-А нереален, однако мы видим, что это НЕ-А не исчезает, ведь без него А логически невозможно. Те не менее: если А существует, HE-А в реальности не должен существовать. Таким образом, НЕ-А существует лишь как возможность, существует «ареально». Формулируя закон противоречия, Аристотель и все логики после него постоянно подчеркивали: не может быть $A$ и HE-А в одном и том же отношении в одно и то же ВРЕМЯ. Мы предлагаем переставить акиенты: в нашей формулировке АРЕАЛЬНОСТЬ - это особое логическое отношение, с помощью которого можно смоделировать естественное Время. Бесконечное множество мгновений Времени является ареальным множеством, поскольку реальность момента Настоямего делает нереальными все другие мгновения этого бесконечного множества. Мы обнаружсиваем, что бесконечным ареальным множеством является также множеством нормировок числовой оси и предлагаем его в качестве модели Времени. В новой модели определяется временной порядок в виде символьной последовательности, где мгновениями являются символь нормировок, представленные в качестве неравных актуально бесконечно малых. Такой подход позволяет обнаружсить на бесконечном множестве Времени периодизаиию, связанную с математической константой е (числом Эйлера). Данный нетривиальный вывод свидетельствует о правомерности предложенной модели.
\end{abstract}

Ключевые слова: время, модель, бесконечность, реальность, нереальность, теория множества.

Научная специальность: 09.00.00 - философские науки. 